\title{
Eficacia de la flutamida en el tratamiento del hirsutismo. Contribución del uso combinado con anticonceptivos orales en mujeres normoandrogénicas
}

\author{
Enzo D evoto C, Lucía Aravena C. \\ Effectiveness of flutamide alone or \\ combined with oral contraceptives in \\ the treatment of hirsutism in women
}

Background: Flutamide is an antiandrogen devoid of other hormonal effects, except for a decrease in the secretion of adrenal androgens such as dehydroepidandrosterone sulphate (DHEA-s) and androstenedione. Aim: To assess the effectiven ess of flutamide in the treatment of hirsutism, used as monotherapy or combined with oral contraceptives (OC). Patients and methods: Women with peripheral hirsutism (defined as the presence of normal serum androgen levels and normal ovulatory menstrual cycles) were assigned to receive flutamide alone (500 mg/day) or flutamide plus an OC (ethynylestradiol $0.03 \mathrm{mg}$ and desogestrel $150 \mu \mathrm{g}$ ). Hirsute with hyperandrogenism (polycystic ovary syndrome) were assigned to receive flutamide plus an OC. The degree of hirsutism was assessed using a clinical score (Moncada) at three, six and twelve months of therapy. Results: Twenty five women with peripheral hirsutism received flutamide alone and 18 receive flutamide plus the contraceptive. Eighteen women with polycystic ovary syndrome were studied. At three months, the reduction in hirsutism was $11.2,15.9$ and $24.7 \%$ in women with peripheral hirsutism receiving flutamide alone or flutamide plus $\mathrm{OC}$ and in hyperandrogenic women receiving flutamide plus OC, respectively. At twelve months, the figures were 57.2, 57.3 and 52.5\% respectively. In hyperandrogenic women, at baseline and three months, serum testosterone levels were 0.96 and $0.42 \mathrm{ng} / \mathrm{ml}$ and serum DHEA-s levels were 2,980 and 1,490 $\mathrm{ng} / \mathrm{ml}$ respectively. No collateral effects of treatment or elevations in serum transaminase levels were observed. Conclusions: Flutamide is effective in the treatment of hirsutism in women with normal or elevated androgen levels. Adding OC did not improve the efficacy of the drug (Rev Méd Chile 2004; 132: 845-52).

(Key Words: Androgen antagonists; Contraceptives, oral, combined; Flutamide; Hirsutism; Polycystic ovary syndrome)

Recibido el 4 de marzo, 2003. Aceptado en versión corregida el 29 de diciembre, 2003.

Facultad de Medicina de la Universidad de Chile, Campus Central. Servicio de Medicina, Sección Endocrinología, Hospital Clínico San Borja-Arriarán. Santiago de Chile.

Correspondencia a: Dr. Enzo Devoto. Hernando de Aguirre

194, Of. 62. Fono: 2314407. E mail: edevoto@vtr.net 
T a terapia farmacológica del hirsutismo pretenLde bloquear el receptor de andrógenos 0 inhibir la 5 alfa reductasa, enzima que transforma testosterona en dihidrotestosterona, en la unidad pilosebácea; esto se logra con antiandrógenos 0 inhibidores específicos de esta enzima como finasteride. En el hirsutismo hiperandrogenémico, además, se espera descender los niveles elevados de andrógenos plasmáticos ${ }^{1}$.

La flutamida, como todo antiandrógeno, desplaza al andrógeno de su receptor impidiendo su acción; carece de otro efecto hormonal estrogénico, androgénico, progestativo, etc, a diferencia de ciproterona y espironolactona ${ }^{5}$.

El único efecto hormonal de la flutamida sería disminuir la secreción de andrógenos suprarrenales (DHEA-S y androstenediona) por inhibición enzimática $^{6,7}, 0$ por disminución del clearance metabólico del cortisol, lo que aumenta su vida media, frena ACTH y por ende disminuye la síntesis de dichos andrógenos ${ }^{8,9}$.

En 22 trabajos publicados entre 1990 y $2000^{4,10-30}$, la flutamida se utilizó como monoterapia en 15 y combinada con anticonceptivo oral (ACO) o agonista de GNRH en 7.

En general los trabajos comprenden períodos cortos de tratamiento de 3 a 4 meses, insuficientes para evaluar una adecuada respuesta $2,3,10,12,17$; un número de pacientes reducido, de 10 o menos $10,11,18,22,23 ;$ y se comunican resultados de terapia de flutamida más $\mathrm{ACO}^{4,13,14,25}$, sin compararla con flutamida sola, no aclarándose en cuánto influye el ACO en la disminución del hirsutismo.

Un ACO constituido por la combinación de estrógeno y gestágeno neutro o muy débilmente androgénico, puede influir favorablemente en el hirsutismo por: a) frenación de LH y descenso de andrógenos ováricos; b) aumento de síntesis hepática de la proteína plasmática transportadora de andrógenos (SHBG) y disminución de la testosterona libre; c) frenación de andrógenos suprarrenales; d) inhibición de la 5 alfa reductasa cutánea ${ }^{31}$.

Con el objeto de clarificar las limitaciones antes señaladas de las publicaciones referentes a la terapia con flutamida en hirsutismo, realizamos un estudio observacional de cohortes para: 1) evaluar la eficacia de flutamida como monoterapia y combinada con ACO; y 2) comprobar si la combinación ACO más flutamida logra un mejor resultado terapéutico en el hirsutismo periférico.

\section{MATERIAL Y MÉTODO}

Las pacientes que consultaron para tratamiento de hirsutismo fueron estudiadas con la metodología utilizada por los autores en publicación anterior ${ }^{3}$. Una vez diagnosticadas se les explicó la terapia a utilizar, señalándoles sus beneficios y riesgos, insistiéndose en el peligro de la falta de masculinización de un feto varón, de ocumir un embarazo bajo antiandrógenos, por lo que sugenimos su combinación con un ACO con gestágeno neutro o muy débilmente androgénico. Dispusimos de flutamida como antiandrógeno, el que ofrecimos a las pacientes enfatizando la baja posibilidad, pero potencial gravedad de su hepatotoxicidad. Sesenta y un pacientes de edad promedio 20,4 años (rango 13-32) dieron su consentimiento informado para ser tratadas con flutamida, 43 eran hirsutas periféricas (normoandrogenémicas, eumenomeicas y ovulatorias), de ellas, 25 por diversos motivos no aceptaron ACO, afirmando no tener vida sexual ni intención de iniciarla próximamente o por utilización de otro método anticonceptivo no hormonal. Dieciocho hirsutas con hiperandrogenemia debido al síndrome de ovario poliquístico (SOP) aceptaron la terapia de flutamida más ACO. Estas pacientes debían cumplir el requisito de DHEA-S normal o sólo ligeramente elevada ( $>3.600$ y $<4.500$ $\mathrm{ng} / \mathrm{ml}$ ). Muy escasas pacientes portadoras de SOP aceptaron ser tratadas sólo con flutamida; informadas del mecanismo de acción prefeńan terapias que bajaran los niveles de andrógenos, regularan ciclos y las protegieran de un eventual embarazo; su número insuficiente impidió constituir el grupo 4 (monoterapia de flutamida en SOP).

Las pacientes se asignaron en 3 grupos: Grupo 1: hirsutas periféricas tratadas con flutamida, $n=25$; Grupo 2: hirsutas periféricas tratadas con flutamida + ACO, n=18; Grupo 3: hirsutas con hiperandrogenemia debida a SOP tratadas con flutamida $+\mathrm{ACO}, \mathrm{n}=18$.

Todas las pacientes debían seguir dieta normal y ejercicio moderado durante el período de observación. De haber retraso menstrual, se exigió prueba de progesterona positiva previo inicio de terapia. Antes de administrar flutamida se evaluó normalidad de la función hepática.

Tratamiento. $250 \mathrm{mg}$ de flutamida cada $12 \mathrm{~h}$. El grupo 2 y 3 recibió además un ACO (etinilestradiol $30 \mathrm{ug}+$ desogestrel $150 \mathrm{ug}$ ) por 21 días, con 7 de descanso. La terapia se inició el primer día de 
un ciclo menstrual espontáneo o inducido por progesterona.

Controles a los 3, 6 y 12 meses, cuantificándose el score de Moncada ${ }^{33}$ efectuado por uno de los autores (ED), se investigó el ciclo menstrual, efectos colaterales de flutamida y del ACO, y se midieron testosterona, DHEA-S, y transaminasas para evaluar riesgo de hepatotoxicidad de la flutamida.

Las determinaciones hormonales por RIA con sus respectivos coeficientes de variación intra e inter ensayo han sido comunicados en publicación anterior de los autores ${ }^{3}$.

Para los objetivos del presente trabajo, en las 61 pacientes se evaluó eficacia de la terapia realizada (objetivo № 1) en base al porcentaje de disminución del score de Moncada. Para cumplir el objetivo № 2 se comparó el grupo de hirsutas periféricas tratadas sólo con flutamida (Grupo 1) con las hirsutas periféricas tratadas con flutamida más ACO (Grupo 2).

Estadística. Se utilizó el test de t de Student para comparación de promedios, aplicado para una cola de distribución y para observaciones pareadas o de muestras con igual varianza según corresponda.

Etica. El trabajo fue analizado por el Comité de Etica del Hospital Clínico San Borja Amiarán, no existiendo objeciones éticas en su diseño y aplicación.

\section{RESULTADOS}

La Tabla 1 muestra clínica y laboratorio de los 3 grupos. Llama la atención en el grupo 3 (SOP) que 3 de 18 pacientes tuvieron ciclos ovulatorios, evaluadas en un nuevo ciclo, éste resultó generalmente anovulatorio. El 50\% de las pacientes del grupo 3 fue eumenorreicas y el otro 50\% presentó amenorrea secundaria u oligomenorrea; todas tenían testosterona elevada y la mayoría DHEA-S normal ( $<3.600 \mathrm{ng} /$ $\mathrm{ml}$ ) excepto 3 cuyo valor máximo fue $4.280 \mathrm{ng} / \mathrm{ml}$.

Las Tablas 2, 3 y 4 analizan la respuesta de los 3 grupos bajo la terapia respectiva, destacando el mayor porcentaje de disminución del hirsutismo en los primeros 3 meses en el grupo 3; a los 12 meses los 3 grupos presentan estimación de disminución muy similares: $57,2 \%, 57,3 \%, 52,5 \%$ en los grupos 1, 2 y 3, respectivamente. Entre el sexto y el duodécimo mes existió un promedio de descenso del score de hirsutismo de 17,3\%. En 2 de las 61 pacientes no hubo disminución del score inicial de hirsutismo al finalizar la terapia.

Tabla 1. Características clínicas y de laboratorio del grupo 1 (flutamida en hirsutas normoandrogenémicas), grupo 2 (flutamida + ACO en hirsutas normoandrogenémicas) y grupo 3 (flutamida + ACO en hirsutas hiperandrogenémicas)

\begin{tabular}{|c|c|c|c|}
\hline & Grupo 1 & Grupo 2 & Grupo 3 \\
\hline $\mathrm{n}$ & 25 & 18 & 18 \\
\hline Edad, años: promedio (rango) & $17,3(13-32)$ & $23,5(17-29)$ & $20,4(14-32)$ \\
\hline IMC promedio (rango) kg/m2 & $22,9(19,1-31,1)$ & $22,9(19,5-33,7)$ & $26,7(19,2-37,5)$ \\
\hline Ciclo menstrual & Eumenorrea: 25 & Eumenorrea: 18 & $\begin{array}{ll}\text { Eumenorrea: } & 9 \\
\text { Oligomenorrea: } & 6 \\
\text { Amenorrea } 2^{\circ}: & 3\end{array}$ \\
\hline $\begin{array}{l}\text { Score de hirsutismo, } \\
\text { puntos: promedio (rango) }\end{array}$ & $11(7-18)$ & $12(8-18)$ & $11,9(8-19)$ \\
\hline $\begin{array}{l}\text { Testosteronemia: } \\
\text { promedio (rango) }\end{array}$ & $0,38(0,18-0,58)$ & $0,36(0,04-0,6)$ & $0,96(0,73-1,4)$ \\
\hline DHEA-S: promedio (rango) & $2.232(800-3.250)$ & $2.245(1.800-3.500)$ & $\begin{array}{l}2.980(2.100-4.280) \\
\text { DHEA-S >3.600: } 3 / 18\end{array}$ \\
\hline $\begin{array}{l}\text { Progesteronemia día } 22 \text { ciclo: } \\
\text { promedio (rango) }\end{array}$ & $7,3(5,2-10)$ & $8,9(4,6-22,4)$ & $\begin{array}{l}1,62(0,63-4,2) \\
\text { Progesteronemia } \\
\text { ovulatoria: } 3 / 18\end{array}$ \\
\hline
\end{tabular}

Valor normal de testosteronemia: hasta $0,6 \mathrm{ng} / \mathrm{ml}$. Valor normal de DHEA-S plasmática: hasta $3.600 \mathrm{ng} / \mathrm{ml}$. Valor ovulatorio de progesteronemia día 22 ciclo: igual $0>4 \mathrm{ng} / \mathrm{ml} 3$. 
La presión arterial no cambió bajo ACO y el peso corporal aumentó sólo en el grupo 2 desde un IMC basal de 22,9 $(19,5-33,7)$ hasta $24,1(19,9-$ $32,4) \mathrm{kg} / \mathrm{m}^{2}$, correspondiente a un incremento promedio de $2,6 \mathrm{~kg}$.

Con respecto a la evolución de la testosterona bajo terapia, no hay variación en el grupo 1 (sólo con flutamida) y hay un leve aumento, estadísticamente significativo en el grupo 2 (flutamida + ACO), dentro del rango normal (Tablas 2 y 3). En el grupo 3, la testosterona basal elevada descendió en $55,2 \%$ al tercer mes de terapia, llegando al año a un descenso del 57,3\%, normalizándose la testosterona en la mayoría (83,3\%); solamente en $3 / 18$ permaneció en valores ligeramente sobre el rango normal de hasta $0,6 \mathrm{ng} / \mathrm{ml}(0,62-0,67-0,7)$.

$\mathrm{Al}$ año de terapia, la DHEA-S disminuyó en 35\% con respecto al basal en el grupo 1 y en $33,4 \%$ en el grupo 2, descensos que ya fueron estadísticamente significativos en el control del tercer mes (Tabla 2 y 3). En el grupo 3, en el control del tercer mes, todas las pacientes tenían DHEA-S normal, alcanzándose disminución de $48 \%$ con respecto al basal al año de terapia (Tabla 4).

No hubo efectos colaterales atribuibles a la flutamida; las transaminasas y fosfatasas alcalinas no sufrieron aumento durante la terapia. Respecto al ACO observamos ligero spotting en los primeros ciclos de tratamiento sin relevancia clínica, y cloasma en 2 pacientes que no obligaron a suspenderlo.

La Tabla 5 compara los resultados de la terapia de hirsutismo del grupo 1 tratado sólo con flutamida versus el grupo 2 tratado con flutamida más ACO, no apreciándose diferencia en el descenso del score a los 12 meses entre ambos grupos.

\section{DisCUSIÓN}

Nuestro universo de 61 hirsutas se compara favorablemente en su $\mathrm{n}$ con trabajos previamente publicados, ya que en 22 trabajos (496 pacientes), 16

\section{Tabla 2. Grupo 1 (flutamida en hirsutas normoandrogenémicas):} evolución del hirsutismo y andrógenos plasmáticos

\begin{tabular}{|c|c|c|c|c|}
\hline & $\begin{array}{l}\text { re de hirsutismo } \\
\text { (puntos) } \\
\overline{\mathrm{x}} \text { (rango) }\end{array}$ & $\begin{array}{l}\text { \% de descenso } \\
\text { del score en } \\
\text { relación al basal }\end{array}$ & $\begin{array}{c}\text { Testosteronemia } \\
\text { ng/ml } \\
\overline{\mathrm{x}}(\text { rango })\end{array}$ & $\begin{array}{c}\text { DHEA-S plasmática } \\
\text { ng/ml } \\
\overline{\mathrm{x}}(\text { rango })\end{array}$ \\
\hline Basal & $1(7-18)$ & - & 0,38 (0,18-0,58) & $2.232(800-3.250)$ \\
\hline 3 meses & $0(7-14)$ & $-11,2 \%$ & $0,41(0,33-0,48)$ & 1.677 (477-2.600) \\
\hline 6 meses & $7 \quad(4-12)$ & $-39,5 \%$ & $0,37(0,11-0,57)$ & $1.400(560-2.700)$ \\
\hline 12 meses & $5 \quad(1-8)$ & $-57,2 \%$ & $0,40(0,10-0,57)$ & $1.448(600-2.800)$ \\
\hline p (3 m vs basal) & & 0,22 (NS) & $0,36(\mathrm{NS})$ & $<0,001$ \\
\hline p (6 m vs basal) & & $<0,001$ & 0,24 (NS) & $<0,001$ \\
\hline $\mathrm{p}$ (12 m vs basal) & & $<0,001$ & 0,47 (NS) & $<0,001$ \\
\hline
\end{tabular}

Tabla 3. Grupo 2 (flutamida + ACO en hirsutas normoandrogenémicas): evolución del hirsutismo y andrógenos plasmáticos

\begin{tabular}{|lcccc|}
\hline & $\begin{array}{c}\text { Score de hirsutismo } \\
\text { (puntos) } \\
\overline{\mathrm{x}} \text { (rango) }\end{array}$ & $\begin{array}{c}\text { \% de descenso } \\
\text { del score en } \\
\text { relación al basal }\end{array}$ & $\begin{array}{c}\text { Testosteronemia } \\
\mathrm{ng} / \mathrm{ml} \\
\overline{\mathrm{x}}(\mathrm{rango})\end{array}$ & $\begin{array}{c}\text { DHEA-S plasmática } \\
\mathrm{ng} / \mathrm{ml} \\
\overline{\mathrm{x}}(\mathrm{rang})\end{array}$ \\
\hline Basal & $12(8-18)$ & - & $0,36(0,04-0,6)$ & $2.245(1.800-3.500)$ \\
3 meses & $10(6-18)$ & $-15,9 \%$ & $0,38(0,10-0,5)$ & $1.680(410-2.800)$ \\
6 meses & $7(4-12)$ & $-45,6 \%$ & $0,37(0,19-0,57)$ & $1.536(390-2.860)$ \\
12 meses & $5(0-10)$ & $-57,3 \%$ & $0,46(0,18-0,6)$ & $1.485(368-2.200)$ \\
$\mathrm{p}(3 \mathrm{~m}$ vs basal) & & $0,21(\mathrm{NS})$ & $0,48(\mathrm{NS})$ & $<0,001$ \\
$\mathrm{p}(6 \mathrm{~m}$ vs basal) & & $<0,001$ & $0,34(\mathrm{NS})$ & $<0,001$ \\
$\mathrm{p}(12 \mathrm{~m}$ vs basal) & & $<0,001$ & $<0,001$ & $<0,001$ \\
\hline
\end{tabular}


tuvieron un $\mathrm{n}$ menor de 22 y solamente 2 , $\mathrm{n}$ de 61 y 65. Nuestras hirsutas fueron tratadas durante un año, período suficiente para evaluar terapia, lo que ocurrió sólo en 10 de las 22 publicaciones citadas.

Respecto a las características clínicas preterapia de las pacientes (Tabla 1), 16,7\% de ciclos ovulatorios del grupo 3 no es excepcional, ya que en SOP $20 \%$ a $30 \%$ de los ciclos pueden ser ovulatorios ${ }^{34}$.

Tradicionalmente se asocia SOP con trastornos menstruales; $50 \%$ de nuestras pacientes tuvo eumenorrea, hecho que no excluye el diagnóstico, ya que hoy se considera el espectro clínico del SOP como muy heterogéneo, comprendiendo desde eumenorrea con hiperandrogenemia hasta amenorrea ${ }^{35}$.

Los hirsutismos periféricos tuvieron estudio de laboratorio que comprobó ovulación y normoandrogenemia (grupos 1 y 2), hechos obligatorios para este diagnóstico ${ }^{3}$.

Las 18 pacientes del grupo 3 presentaron elevación de la testosterona total y solamente $3 / 18$ un discreto aumento de la DHEA-S; este pequeño porcentaje de pacientes debería llamar la atención, ya que la DHEA-S se encuentra elevada en $50 \%$ del SOP, lo que ha sido señalado en la literatura $^{36}$ y corroborado por nosotros ${ }^{37}$. En el presente trabajo esto se explica por el criterio de exclusión adoptado, de no incluir en el grupo con hiperandrogenemia las pacientes con DHEA-S mayor de $4.500 \mathrm{ng} / \mathrm{ml}$.

La DHEA-S muy elevada puede contribuir a aumentar el nivel plasmático de testosterona actuando como sustrato a interconvertirse en testosterona. Si no hubiésemos eliminado esta variable (DHEA-S muy elevada) mediante el criterio de exclusión antes señalado, sería difícil de interpretar si el descenso de la testosterona se debía a la supresión ovárica generada por el ACO, o al menor nivel de DHEA-S logrado por la frenación androgénica suprarrenal ejercida por la flutamida y el ACO.

La evaluación de la terapia fue realizada por un método semiobjetivo, score de Monca$\mathrm{da}^{33}$, sujeto a considerable variación interobservador e incluso intraobservador ${ }^{38}$, motivo

Tabla 4. Grupo 3 (flutamida + ACO en hirsutas con hiperandrogenemia): evolución del hirsutismo y andrógenos plasmáticos

\begin{tabular}{|c|c|c|c|c|}
\hline & $\begin{array}{l}\text { Score de hirsutismo } \\
\text { (puntos) } \\
\overline{\mathrm{x}} \text { (rango) }\end{array}$ & $\begin{array}{l}\% \text { de descenso } \\
\text { del score en } \\
\text { relación al basal }\end{array}$ & $\begin{array}{c}\text { Testosteronemia } \\
\text { ng/ml } \\
\overline{\mathrm{x}}(\text { rango })\end{array}$ & $\begin{array}{c}\text { DHEA-S plasmática } \\
\text { ng/ml } \\
\bar{x}(\text { rango })\end{array}$ \\
\hline Basal & $11,9 \quad(8-19)$ & - & $0,96(0,73-1,4)$ & $2.980(2.100-4.280)$ \\
\hline 3 meses & $8 \quad(6-15)$ & $-24,7 \%$ & $0,51(0,22-0,52)$ & $1.560(992-3.400)$ \\
\hline 6 meses & $7 \quad(5-15)$ & $-41,9 \%$ & $0,43(0,11-0,6)$ & $1.498(980-3.375)$ \\
\hline 12 meses & $(2-13)$ & $-52,5 \%$ & $0,41(0,11-0,7)$ & $1.548(700-3.800)$ \\
\hline p (3 m vs basal) & & $<0,001$ & $<0,001$ & $<0,001$ \\
\hline $\mathrm{p}$ (6 m vs basal) & & $<0,001$ & $<0,001$ & $<0,001$ \\
\hline $\mathrm{p}$ (12 m vs basal) & & $<0,001$ & $<0,001$ & $<0,001$ \\
\hline
\end{tabular}

Tabla 5. Resultados del tratamiento del hirsutismo con flutamida (grupo 1) vs flutamida + ACO (grupo 2)

\begin{tabular}{|lrcccccc|}
\hline & \multicolumn{2}{c}{$\begin{array}{c}\text { Score } \\
\text { (puntos) }\end{array}$} & $\begin{array}{c}\text { \% de descenso } \\
\text { del score en } \\
\text { relación al basal }\end{array}$ & $\begin{array}{c}\text { Score } \\
\text { (puntos) }\end{array}$ & $\begin{array}{c}\text { \% de descenso } \\
\text { del score en } \\
\text { relación al basal }\end{array}$ & $\mathrm{p}$ \\
\hline Basal & 11 & $(7-18)$ & - & 12 & $(8-18)$ & - & \\
3 meses & 10 & $(7-14)$ & $-11,2 \%$ & 10 & $(6-18)$ & $-15,9 \%$ & 0,22 (NS) \\
6 meses & 7 & $(4-12)$ & $-39,5 \%$ & 7 & $(4-12)$ & $-45,6 \%$ & 0,19 (NS) \\
12 meses & 5 & $(1-8)$ & $-57,3 \%$ & $5(0-10)$ & $-57,3 \%$ & 0,42 (NS) \\
\hline
\end{tabular}


por el cual preferimos que la evaluación basal y los controles bajo terapia del score de hirsutismo fueran realizados por un solo observador, criterio ya adoptado por nosotros en comunicaciones anteriores 3,37 . Una limitante a considerar es el hecho que el observador no estaba ciego a la terapia.

En los 3 grupos el mayor porcentaje de descenso del hirsutismo se observó al sexto mes; la ganancia adicional de disminución lograda entre los 6 y 12 meses, refuerza la necesidad de un tratamiento prolongado ${ }^{3}$.

En los 3 grupos la terapia fue eficaz, obteniéndose un promedio de disminución del hirsutismo a los 12 meses, de 55,7\%, coincidente con 53,7\% que señala la literatura ${ }^{4,10-30}$. Este promedio de disminución y el bajo porcentaje de fracaso del tratamiento $(3,3 \%)$ confirma su eficacia.

El mayor descenso del score en el grupo hiperandrogenémico al tercer mes, puede deberse a la importante reducción de los andrógenos plasmáticos. Carr obtuvo un resultado similar y señaló la conveniencia en las hirsutas hiperandrogenémicas de la frenación androgénica al inicio de la terapia, lo que podría mejorar la adherencia al tratamiento 39 .

En la literatura hay divergencias referente al efecto de flutamida en testosterona plasmática; sin cambios ${ }^{10-12,14,16,19,24}$ o descenso $15,20,22,23,26$. En el grupo 1 la testosterona no fue influida por la flutamida y el leve aumento en el grupo 2 se podría interpretar debido al incremento de SHBG por ACO, que aumenta la testosterona total. El descenso ya señalado de testosterona en el grupo hiperandrogenémico se explicaría por la acción frenadora de LH del ACO, aunque algunos postulan una posible acción de flutamida mediante su influencia en la pulsatilidad de $\mathrm{LH}^{40}$

El descenso de la DHEA-S en el grupo 1, atribuible a la flutamida, coincide con unos ${ }^{12,14,16}$ y difiere de otros ${ }^{4,11,19}$. Habríamos esperado un mayor descenso en el grupo 2, ya que clásicamente se señala que el ACO frena la secreción de DHEA-S ${ }^{31}$, aunque algunos autores han comunicado la falta de cambios del nivel de DHEA-S bajo $\mathrm{ACO}^{39,41}$.
La flutamida presenta reacciones adversas, como hepatotoxicidad en $1 \%$, la que no constatamos en este grupo de pacientes.

Los efectos colaterales del ACO fueron muy discretos, sin significación clínica y están dentro de lo esperado para este tipo de combinación (etinilestradiol/desogestrel) ${ }^{42}$

No logramos comprobar que el agregado del ACO a la flutamida mejorara su efecto terapéutico en hirsutismo periférico, pues no hubo diferencia estadísticamente significativa en el score final obtenido en grupo 1 versus grupo 2. Llama la atención la inexistencia de este efecto aditivo terapéutico, ya que con ACO, en forma exclusiva, se logran descensos del score de $25 \%{ }^{42}, 29 \%{ }^{43}$ y $40 \%{ }^{44}$, aunque otros dicen que este efecto del ACO es muy leve 0 inexistente ${ }^{1}$. Gregoriou ${ }^{43}$ en contraste con nuestros resultados, demostró este efecto aditivo en terapia de hirsutismo con otro antiandrógeno (espironolactona), combinado con el mismo ACO por nosotros utilizado (etinilestradiol/desogetrel), comparando esta combinación con dicho ACO utilizado como monoterapia.

Como ya señalamos, uno de los posibles mecanismos de acción del ACO en terapia de hirsutismo es aumentar la SHBG y disminuir la testosterona libre. Aunque no las determinamos, Culberg ${ }^{42}$ demostró con etinilestradiol/desogestrel un aumento de 5 veces la SHBG y disminución de la testosterona libre, lo que debería haber ocurrido en nuestras pacientes. Si estos cambios bioquímicos tuvieran un importante significado clínico, debieron traducirse en mayor disminución del score de hirsutismo en el grupo 2 comparado con el 1. A similares resultados llegaron Elkind-Hirsch y Azziz, quienes también discuten la real importancia clínica de dicho cambio de laboratorio ${ }^{41,45}$.

La flutamida se demostró eficaz en esta serie, y con las precauciones ya señaladas con respecto a función hepática, segura en terapia de hirsutismo.

El agregado del ACO no mejoró la eficacia terapéutica de la flutamida, pero esto no descarta su uso en terapia de todo hirsutismo por su rol de prevenir un embarazo bajo antiandrógenos, además regular el ciclo menstrual y proteger al endometrio del desbalance estrógeno progesterona en el hirsutismo hiperandrogenémico anovulatorio. 


\section{REFERENCIAS}

1. RITTMASTER RS. Treating hirsutism. Endocrinologist 1993; 3: 211-8.

2. Devoto E, Aravena L Utilidad terapéutica de los antiandrógenos. Rev Méd Chile 1993; 121: 118390.

3. Devoto E, Aravena L, Ríos R. Tratamiento del hirsutismo con espironolactona y con espironolactona más dexametasona. Rev Méd Chile 2000; 128: 868-75.

4. Cusan L, Dupont A, Bélanger A, Tremblay RR, Manhes G, Labrie F. Treatment of hirsutism with the pure antiandrogen flutamide. J Am Acad Dermatol 1990; 23: 462-9.

5. LABRIE F. Mechanism of action and pure antiandrogen properties of flutamide. Cancer 1993; 72: 3816-27.

6. Brogden RN, Cussold SP. Flutamide. A preliminary review of its pharmacodynamic and pharmacokinetic properties and therapeutic efficacy in advanced prostatic cancer. Drugs 1989; 38: 185-203.

7. Ayub M, LeveL MJ. Inhibition of rat testicular 17 hydroxylase and 17,29 lyase activities by anti-androgens (Flutamide, Hydroxyflutamide, RU23908, Cyproterone acetate) in vitro. J Steroid Biochem 1987; 28: 43-7.

8. Funushima DK, Levin J, Krean J, Freed SZ, Whitmore WF, Heluman L et aL. Effect of Flutamide on cortisol metabolism. J Clin Endocrinol Metab 1978; 47: 788-91.

9. Charmandari E, Johnston A, Honour JW, Brook CG, HindMARSH PC. Treatment with flutamide decreases cortisol clearance: implications for therapy in congenital adrenal hyperplasia. J Pediatr Endocrinol Metab 2002; 15: 235-9.

10. Marcondes JAM, Minnani SL, Luthold W, WajchnBERG BL, SAMOJLK E, KirSchner MA. Treatment of hirsutism in women with flutamide. Fertil Steril 1992; 57: 543-7.

11. Couzinet B, Pholsena M, Young J, Schaison G. The impact of a pure anti - androgen (flutamide) on LH, FSH, androgens and clinical status in idiopathic hirsutism. Clin Endocrinol 1993; 39: 157-62.

12. Fruzzetti F, De Lorenzo D, Ricci C, Fioretti P. Clinical and endocrine effects of flutamide in hyperandrogenic women. Fertil Steril 1993; 60: 806-13.

13. Ciotta L, Cianci A, Marletta E, Pisana L, Agliano A, Palumbo G. Treatment of hirsutism with flutamide and low-dosage oral contraceptive in polycystic ovarian disease patients. Fertil Steril 1994; 62: 1129-35.

14. Cusan L, Dupont A, Gómez JL, Tremblay RR, Labrie F. Comparison of flutamide and spironolactone in the treatment of hirsutism: a randomized controlled trial. Fertil Steril 1994; 61: 281-7.

15. Moghetti $\mathrm{P}$, Castello R, Negri C, Tosi F, Magnani CM, Fontanarosa MC et al. Flutamide in the treatment of hirsutism: long-term clinical effects endocrine changes, and androgen receptor behavior. Fertil Steril 1995; 64: 511-7.

16. Dodin S, Faure N, Cédrin I, Méchain C, TurcotLeMAY L, GuY J ET AL. Clinical efficacy and safety of low-dose flutamide alone and combined with an oral contraceptive for the treatment of idiopathic hirsutism. Clin Endocrinol 1995; 43: 57582.

17. Diamanti-Kandarakis E, Mitrakou A, Hennes MMI, Piatanissiotis D, Kakias N, Spina J et al. Insulin sensitivity and antiandrogenic therapy in women with polycystic ovary syndrome. Metabolism 1995; 44: 525-31.

18. Moghetti P, Tosi F, Castello R, Nagnani CM, Negri C, BRUN E ET AL. The insulin resistance in women with hyperandrogenism is partially reversed by antiandrogen treatment: evidence that androgens impair insulin action in women. J Clin Endocrinol Metab 1996; 81: 952-60.

19. MüderRis II, Bayram F, Sahin Y, Kelestimur F, Tutus A, Ayata D. The efficacy of $250 \mathrm{mg} /$ day flutamide in the treatment of patients with hirsutism. Fertil Steril 1996; 66: 220-2.

20. Falsetti L, De Fusco D, Eleftheriou G, Rosina B. Treatment of hirsutism by finasteride and flutamide in women with polycystic ovary syndrome. Gynecol Endocrinol 1997; 11: 251-7.

21. Müderris II, Bayram F, Sahin Y, Kelestimur F. A comparision between two doses of flutamide $(250 \mathrm{mg} / \mathrm{d}$ and $500 \mathrm{mg} / \mathrm{s}$ ) in the treatment of hirsutism. Fertil Steril 1997; 68: 644-7.

22. De Leo V, la Marca A, Lanzetta D, Cariello PL, D'Antona D, Morgante G. Effects of flutamide on pituitary and adrenal responsiveness to corticotrophin releasing factor (CRF). Clin Endocrinol 1998; 49: 85-9.

23. De Leo V, Lanzetta D, D’Antona D, la Marca A, Morgante G. Hormonal effects of flutamide in young women with polycystic ovary syndrome. J Clin Endocrinol Metab 1998; 83: 99-102. 
24. Fruzzetti F, Bersi C, Parrini D, Ricci C, Genazzani AR. Treatment of hirsutism: comparision between different antiandrogens with central and peripheral effects. Fertil Steril 1999; 71: 445-51.

25. Pazos F, Escobar-Morreale HF, Balsa J, Sancho JM, Varela C. Prospective randomized study comparing the long-acting gonadotropin-releasing hormone agonist triptorelin, flutamide, and cyproterone acetate, used in combination with an oral contraceptive, in the treatment of hirsutism. Fertil Steril 1999; 71: 122-8.

26. Venturoli S, Marescalchi O, Colombo FM, Macrew B, Ravaiol B, Bagnoli A et al. A prospective randomized trial comparing low dose flutamide, finasteride, ketoconazole, and cyproterone acetate-estrogen regimens in the treatment of hirsutism. J Clin Endocrinol Metab 1999; 84: 1304-10.

27. Moghetti $\mathrm{P}$, Tosi F, Tosti A, Negri C, Miscial C, PerRone F ET al. Comparison of spironolactone, flutamide, and finasteride efficacy in the treatment of hirsutism: a randomized, double blind, placebo-controlled trial. J Clin Endocrinol Metab 2000; 85: 89-94.

28. Ibáñez L, Potau N, Marcos MV, De Zegher F. Treatment of hirsutism, hyperandrogenism, oligomenorrhea, dyslipidemia, and hyperinsulinism in nonobese, adolescent girls: effect of flutamide. J Clin Endocrinol Metab 2000; 85: 3251-5.

29. De Leo V, Fulghesu AM, la Marca A, Morgante G, Pasqui L, TALuni B et al. Hormonal and clinical effects of GNRH agonist alone, or in combination with a combined oral contraceptive or flutamide in women with severe hirsutism. Gynecol Endocrinol 2000; 14: 411-6.

30. MüdeRris II, Bayram F, Güven M. Treatment of hirsutism with lowest-dose flutamide $(62.5 \mathrm{mg} /$ day). Gynecol Endocrinol 2000; 14: 38-41.

31. AzZIZ R, GAY F. The treatment of hyperandrogenic with oral contraceptives. Sem Reprod Endocrinol 1989; 7: 246-54.

32. Wysowski DK, Fourcroy JL. Flutamide hepatotoxicity. J Urol 1996; 155: 209-12.

33. Moncada Lorenzo E. Familial study of hirsutism. J Clin Endocrinol Metab 1970; 31: 556-64.

34. GoLdZIEHER JW, AXeLROD LR. Clinical and biochemical features of polycystic ovarian disease. Fertil Steril 1963; 14: 631-53.
35. Carmina E, Lobo RA. Polycystic ovaries in hirsute women with normal menses. Am J Med 2001; 111: 602-6.

36. Azziz R. Hypothalamic pituitary adrenal axis dysfunction in PCOS. In: Azziz R, Nestler JE, Dewailly $\mathrm{D}$ ed. Androgens excess disorders in women. Lippincott - Raven publishers. Philadelphia. 1997; 395-402.

37. Devoto E, Aravena L Hirsutismo: experiencia en diagnóstico y tratamiento en el período 19701995. Rev Chil Obstet Ginecol 1995; 60: 291-301.

38. BARTH JH. How robust is the methodology for trials of therapy in hirsute women? Clin Endocrinol 1996; 45: 379-80.

39. Carr BR, Breslau NA, Givens C, Byrd W, BernettHamm C, Marshburn PB. Oral contraceptives pills, gonadotropin-releasing hormone agonist, or use in combination for treatment of hirsutism: a clinical research center study. J Clin Endocrinol Metab 1995; 80: 1169-78.

40. SiR T. ¿Modulan los andrógenos la secreción de hormona luteinizante en mujeres? Rev Méd Chile 1997; 125: 710-8.

41. EiKIND-Hirsh KE, ANania C, Mack M, MaLnak R. GNRH agonist plus oral contraceptive «ad back» therapy stimulate sex hormone binding globulin, and lowers free testosterone in hirsute women with ovarian hyperandrogenism. Fertil Steril 1995; 63: 970-8.

42. Culberg G, Hamberger L, Mattson L, Mobacken H, Samsioe G. Effects of a low dose desogestrelethinylestradiol combination on hirsutism, androgen and sex hormone binding globulin in women with a polycystic ovary syndrome. Acta Obstet Gynecol Scand 1985; 64: 195-202.

43. Gregoriou O, Bakas P, Konidaris S, Papadias K, Mathiopoulos D, Creatsas G. The effect of combined oral contraception with or without spironolactone on bone mineral density of hyperandrogenaemic women. Human Reproduction 1993; 8: 1807-12.

44. Porcile A, Galardo E. Long-term treatment of hirsutism: desogestrel compared with cyproterone acetate in oral contraceptives. Fertil Steril 1991; 55: 877-81.

45. Azziz R, Ochoa TM, Bradiey EL, Downing Potter $\mathrm{H}$, Bоотs LR. Leuprolide and estrogen versus oral contraceptive pills for the treatment of hirsutism: a prospective randomized study. J Clin Endocrinol Metab 1995; 80: 3406-8. 